\title{
ENFERMAGEM NEONATOLÓGICA: CONHECIMENTO DE ALUNOS DE GRADUAÇÃO EM ENFERMAGEM*
}

\author{
Masuco Naganuma** \\ Massae Noda Chaud** \\ Eliana Moreira Pinheiro***
}

NAGANUMA, M.; CHAUD, M.N.; PINHEIRO, E.M. Enfermagem neonatológica: conhecimento de alunos de graduação em enfermagem. Rev.latino-am.enfermagem, Ribeirão Preto, v. 7, n. 4, p. 75-81, outubro 1999.

O presente estudo foi realizado com alunos dos cursos de graduação em Enfermagem dos Municípios de São Paulo e de Guarulhos, com a finalidade de avaliar o seu conhecimento sobre enfermagem neonatológica e contribuir, se necessário, para a reformulação do programa. Dessa forma, poderíamos aprimorar o conhecimento do enfermeiro sobre os cuidados do recémnascido a termo. Foram selecionados os aspectos do conteúdo teórico concernentes aos cuidados mais comuns na unidade de neonatologia para elaborar um questionário estruturado, que foi utilizado como instrumento de mensuração da variável. Observouse que o nivel de conhecimento teórico dos estudantes sobre o tema era regular.

UNITERMOS: enfermagem materno-infantil, educação

\section{INTRODUÇÃO}

O presente estudo é resultado de uma pesquisa realizada entre alunos dos cursos de graduação em enfermagem dos Municípios de São Paulo e de Guarulhos sobre o conhecimento das especificidades do recémnascido e respectivos cuidados de enfermagem indispensáveis à sua saúde e bem-estar.

A enfermeira da unidade de neonatologia assiste a uma clientela com características e peculiaridades especiais, no caso o recém-nascido e sua mãe, num momento de grande vulnerabilidade, que exige conhecimentos específicos para dar-lhes a devida assistência.

Desde o primeiro minuto de vida, o recémnascido deve ser observado com extremo cuidado, pois ele é particularmente sensível às infecções, por não ter resistência e meios de defesa (PIZZATO \& POIAN, 1988).

A fim de garantir ao recém-nascido adaptação satisfatória à vida extra-uterina, torna-se necessária, entre outros procedimentos, uma observação cuidadosa que compreende: (1) avaliação inicial realizada através do Índice de Apgar; (2) avaliação transicional durante o período da reatividade e (3) avaliação periódica efetiva por meio do exame físico sistemático. Para a realização dessas atividades, é necessário que o enfermeiro tenha conhecimento dos dados normais esperados em cada fase do período pós-natal, para que possa reconhecer qualquer intercorrência e intervir, adequadamente, para que o neonato tenha desenvolvimento satisfatório (WHALEY \& WONG, 1989).

O recém-nascido necessita de cuidados que possibilitem sua adaptação ao mundo exterior; portanto as atenções a ele dispensadas na unidade de neonatologia, nos primeiros momentos, podem ser decisivas para a sua vida futura.

Há duas décadas, a SÃO PAULO - SES (1976), entre outras diretrizes, recomendava que o serviço de Enfermagem no berçário de recém-nascidos permanecesse sob supervisão constante de um enfermeiro com treinamento especial na assistência ao neonato e que as equipes de Enfermagem e Médica desenvolvessem trabalho conjunto. Determinava, outrossim, que grande parte da responsabilidade no cuidado do recém-nascido e no controle das infecções adquiridas coubesse à equipe de enfermagem. Complementando, ressaltava que para a assistência adequada ao recém-nascido no berçário é necessário um serviço organizado, pressupondo uma estrutura com profissionais altamente qualificados.

A formação de profissionais qualificados tem sido a preocupação constante no exercício de nossa

\footnotetext{
* Dados extraídos da Tese de Doutorado em Enfermagem Materna e Infantil apresentada em 1993 à Escola Paulista de Medicina

** Professor Adjunto da Universidade Federal de São Paulo (UNIFESP)

*** Professor Assistente da Universidade Federal de São Paulo (UNIFESP)
} 
docência. A aplicação do conhecimento da teoria para a prática deve ser aprendida pelo aluno. Para tanto, o professor entre outras ações deve facilitar, estimular e encorajar a aquisição de habilidades do educando.

Da crença nas considerações expostas, assim como nas reflexões coletivas dos professores, preocupados com o ensino de enfermagem neonatológica, resultou este trabalho que contempla interesses e questões surgidas ao longo do exercício profissional.

Assim, o presente estudo tem como objetivo avaliar o conhecimento sobre os conteúdos teóricos de Enfermagem Neonatológica de alunos dos cursos de graduação em enfermagem dos Municípios de São Paulo e de Guarulhos, contribuir para uma discussão mais ampla entre docentes e enfermeiros da área sobre a assistência ao recém-nascido a termo.

\section{METODOLOGIA}

\section{Amostra}

A amostra desta pesquisa é composta de alunos de nove cursos de graduação em Enfermagem, sendo 290 alunos de sete escolas da rede privada e 67 alunos de duas escolas da rede pública, totalizando 357 estudantes que já haviam recebido os conteúdos teóricos de enfermagem neonatológica.

\section{VARIÁVEL DO ESTUDO}

A variável de estudo é o conhecimento de alguns aspectos do conteúdo teórico de Enfermagem Neonatológica de alunos dos cursos de graduação em Enfermagem, doravante denominada "conhecimento teórico sobre enfermagem neonatológica".

Para a seleção dos aspectos da variável conhecimento teórico sobre enfermagem neonatológica - foram solicitados aos cursos de graduação em enfermagem dos municípios selecionados, os programas de enfermagem neonatológica com a finalidade de detectar quais os aspectos comuns oferecidos para a formação dos enfermeiros nesta área de conhecimento. Por meio do estudo minucioso desses programas, elaborou-se uma relação dos temas de enfermagem neonatológica desenvolvidos pelos cursos. Essa relação, analisada criticamente, tendo por base os conhecimentos mínimos de enfermagem neonatológica necessários à formação do profissional, mostrou a necessidade de incluir assuntos não discriminados nos programas.

Elaborada nova relação de temas, esta foi ajuizada por duas enfermeiras docentes de enfermagem neonatológica, com ampla experiência na área, que sugeriram acrescentar outros aspectos, ampliando-a. Reelaborada, a lista de conteúdos foi cuidadosamente analisada e decidiu-se eliminar alguns procedimentos básicos de enfermagem ministrados em disciplinas que antecedem a enfermagem neonatológica. Esta tarefa culminou com a definição dos conteúdos teóricos mínimos de enfermagem neonatológica que um curso de graduação deve oferecer aos seus alunos. Assim, esses aspectos foram agrupados em cuidados imediatos, cuidados mediatos e outros cuidados de rotina ao recémnascido a termo, de acordo com SEGRE \& MARINO (1991); VIEGAS \& MONETTI (1989) e VAZ et al. (1989).

Complementando o estudo considerou-se pertinente a inclusão da variável percepção do aluno sobre a suficiência do conteúdo teórico de Enfermagem Neonatológica ministrado e o grau de aproveitamento.

\section{INSTRUMENTO DA PESQUISA}

O instrumento utilizado para coleta de dados foi o questionário constituído de 19 perguntas estruturadas, abordando conteúdos sobre cuidados imediatos, mediatos e outros cuidados de rotina ao recém-nascido a termo e percepção sobre os aspectos já mencionados.

\section{COLETA DE DADOS}

O questionário foi aplicado entre alunos dos cursos de graduação em enfermagem dos municípios de São Paulo e Guarulhos, que haviam tido os conteúdos de enfermagem neonatológica, no período de 03 a 20 de agosto de 1992.

Foi entregue junto com o questionário a Carta de Apresentação e Termo de Consentimento (Anexo) com informações e termo de consentimento sobre o trabalho, anexo, solicitando a participação do aluno e orientandoo sobre o modo de proceder para responder ao instrumento, assim como assegurando seu anonimato.

\section{RESULTADOS}

O estudo descritivo das variáveis é apresentado em números absoluto e relativo (porcentagem) limitandose aos aspectos considerados essenciais ao conhecimento sobre alguns aspectos dos cuidados imediatos (Tabela 1), mediatos (Tabela 2) e outros cuidados de rotina (Tabela 3) ao recém-nascido a termo e à percepção do aproveitamento e da suficiência do ensino teórico de Enfermagem Neonatológica (Tabela 4). 
Tabela 1 - Conhecimento dos alunos de graduação em Enfermagem sobre cuidados imediatos ao recém-nascido a termo

\begin{tabular}{|c|c|c|c|c|}
\hline \multicolumn{3}{|c|}{ Cuidados imediatos } & \multicolumn{2}{|c|}{ Freqüuência } \\
\hline \multicolumn{2}{|r|}{ Aspectos } & Respostas & Absoluta & Relativa \% \\
\hline BOLETIM & Mormentos da feitura & certa & 273 & 77,0 \\
\hline \multirow{3}{*}{$\mathrm{DE}$} & & errada & 82 & 23,0 \\
\hline & Finalidade & certa & 151 & 42,8 \\
\hline & & errada & 202 & 57,2 \\
\hline $\mathrm{APGAR}$ & Sinais para avaliação & $\begin{array}{l}\text { certa } \\
\text { errada }\end{array}$ & $\begin{array}{l}217 \\
134\end{array}$ & $\begin{array}{l}61,8 \\
382\end{array}$ \\
\hline \multirow{4}{*}{$\begin{array}{l}\text { MANUTENÇÃO } \\
\text { DA } \\
\text { TEMPERATURA }\end{array}$} & Tipo de campo para recepcão do & certa & 253 & 71.1 \\
\hline & recém-nascido & errada & 103 & 28,9 \\
\hline & Mecarismo da perda de calor & certa & 65 & 18,9 \\
\hline & & errada & 279 & 81,1 \\
\hline \multirow{3}{*}{\multicolumn{2}{|c|}{$\begin{array}{l}\text { Desobstrução das vias aéreas superiores: } \\
\text { ordem de aspiração } \\
\text { Constituição dos vasos do coto umbilical }\end{array}$}} & certa & 173 & 48,8 \\
\hline & & errada & 181 & 51,2 \\
\hline & & certa & 153 & 43,4 \\
\hline & & errada & 200 & 56,6 \\
\hline \multirow[t]{4}{*}{ CREDEIZAÇÃO } & Tipo de substância & certa & 292 & 82,3 \\
\hline & & errada & 63 & 17,7 \\
\hline & Finalidade & certa & 324 & 91,1 \\
\hline & & errada & 32 & 8,9 \\
\hline
\end{tabular}

Nota: A soma das treqüèncias absolutas de cada aspecto não totalıza 35 / alunos (total da amostra) em vista de alguns não terem respondido todas as questões

Fonte: Alunos de Graduação em enfermagem dos Municípios de São Paulo e de Guarulhos

Na Tabela 1 verifica-se que $77,0 \%$ dos alunos responderam corretamente sobre os momentos, $1^{\circ}$ e $5^{\circ}$ minutos da feitura do Boletim de Apgar; $61,8 \%$ dos alunos referiram acertadamente freqüência cardíaca, esforço respiratório, tônus muscular, irritabilidade reflexa e cor da pele como sinais para avaliação da vitalidade do recém-nascido e apenas $42,8 \%$ da amostra sabiam que a finalidade do Boletim de Apgar é avaliar a vitalidade do recém-nascido. A avaliação das respostas foi fundamentada em trabalhos de SEGRE \& MARINO (1991); WHALEY \& WONG (1989); PIZZATO \& POIAN (1988) e RAMOS (1978).

Considerou-se o conhecimento sobre o Boletim de Apgar insuficiente, pois os alunos sabiam os momentos de sua realização e quais os sinais a serem pesquisados, mas não informaram o objetivo dessas ações. Infere-se que, provavelmente, suas ações relativas à aplicação do Boletim de Apgar não estão cientificamente fundamentadas, pois o enfermeiro para desempenhá-las adequadamente deve saber quando executar, o que verificar e para que realizar tais procedimentos.

Essa mesma situação se repetiu no conhecimento sobre manutenção da temperatura, na questão, relativa ao tipo de campo utilizado para recepcionar o recémnascido. Número razoável $(71,1 \%)$ de respondentes sabem que para evitar o resfriamento do recém-nascido, este deve ser envolto em campo esterilizado e aquecido conforme SEGRE (1991); VIEGAS \& MONETTI (1989); RAMOS (1978). Ao responder sobre mecanismos da perda de calor do recém-nascido, apenas 18,9\% apontaram que é através de condução, evaporação, convecção e radiação. A manutenção do calor corporal do recém-nascido é um dos aspectos da assistência de enfermagem. Para evitar a perda de calor o recém-nascido deve ser assistido em sala sem corrente de ar, ser recebido em campo estéril e aquecido, colocado em superfície aquecida e mantido aquecido durante o transporte, de acordo com os autores retro mencionados. Este resultado foi considerado insatisfatório, visto que o enfermeiro necessita conhecer quais os fatores que contribuem para a perda de calor, sua conseqüência e, portanto, intervir fundamentado em conhecimento científico.

Os dados relativos à ordem de aspiração para desobstrução das vias aéreas superiores do recém-nascido, expressos na Tabela 1, mostram que menos da metade $(48,8 \%)$ da amostra respondeu corretamente que a ordem é boca, faringe e narinas.

MARINHO (1981); SEGRE \& MARINO (1991) recomendam iniciar a aspiração sempre pela boca e nunca pelas narinas. Caso a aspiração não obedeça a ordem referida e se esta iniciar-se pelas fossas nasais, pode, por estimulação reflexa, provocar aspiração do material da faringe, causando problemas respiratórios. Neste sentido MARINHO (1981); RAMOS et al. (1978) salientam que a não obediência para obstrução das vias aéreas superiores tem possibilidade de provocar, reflexamente, inspiração, levando mucosidade da boca para os pulmões. Desse modo, este procedimento aparentemente simples se não executado corretamente pode acarretar prejuízos ao 
recém-nascido, portanto espera-se que este conhecimento seja do domínio de todos os alunos que tiveram o aprendizado em enfermagem neonatológica.

Na Tabela 1, sobre constituição dos vasos do coto umbilical, responderam corretamente - uma veia e duas artérias - menos da metade $(43,4 \%)$ da amostra. O coto umbilical do recém-nascido deve ser inspecionado para verificar a possibilidade de artéria única; caso esta ocorra, o recém-nascido pode apresentar outras malformações, muitas vezes não evidentes no momento do nascimento, conforme CASANOVA (1991).

$\mathrm{O}$ resultado referente à substância utilizada na credeização, exposto na Tabela 1 , mostra que a maioria $(82,3 \%)$ dos informantes sabe que o colírio utilizado é de nitrato de prata a $1 \%$. Quanto à finalidade da credeização, a quase totalidade $(91,1 \%)$ dos alunos respondeu corretamente que é para prevenir conjuntivite gonocócica. Conforme WHALEY \& WONG (1989), o tratamento profilático contra Neisseria Gonorrhoeae, que pode causar cegueira em criança nascida de mãe com gonorréia, é realizado pela instilação de uma gota de solução de nitrato de prata em cada olho. O índice de respostas foi bastante favorável, e pode ser devido ao fato de o tema ser tratado tanto nos conteúdos de Enfermagem Neonatológica como no de Enfermagem Obstétrica.

No que concerne ao ensino teórico sobre os cuidados mediatos ao recém-nascido, foram selecionadas três questões consideradas relevantes cujos resultados estão expostos a seguir.

Tabela 2 - Conhecimento dos alunos de Graduação em Enfermagem sobre os cuidados mediatos ao recém-nascido a termo

\begin{tabular}{lccc}
\hline \multicolumn{1}{c}{ Cuidados mediatos } & \multicolumn{2}{c}{ Freqüência } \\
\hline \multicolumn{1}{c}{ Aspectos } & Respostas & Absoluta Relativas \% \\
\hline Sinais de mumificação do & certa & 249 & 69,9 \\
coto umbilical & errada & 107 & 30,1 \\
Período de troca de filtro & certa & 40 & 11,3 \\
da incubadora & errada & 315 & 88,7 \\
Limite máximo de tempo & certa & 89 & 25,4 \\
para eliminação de & errada & 262 & 74,6 \\
mecônio & & & \\
\hline
\end{tabular}

Nota: A soma das freqüências absolutas de cada aspecto não totaliza 357 alunos (total da amostra) em vista de alguns não terem respondido todas as questões

Fonte: Alunos de Graduação em enfermagem dos Municípios de São Paulo e de Guarulhos

A resposta certa - coto umbilical escuro e seco sobre sinais de mumificação do coto, foi indicada por número satisfatório $(69,9 \%)$ de alunos. Entretanto, considera-se que os $30,1 \%$ que deram respostas incorretas constituíram universo expressivo para o tipo de conhecimento avaliado que é de fácil identificação na prática.
A respeito do conhecimento sobre período de troca de filtro da incubadora, apenas $11,3 \%$ dos estudantes sabem que o procedimento deve ser feito a cada 90 dias, número este considerado baixo e por isso preocupante.

Conforme manual de instrução da fábrica, o ar que entra na incubadora passa por um sistema de microfiltragem que retém microorganismos, possibilitando ao recém-nascido receber ar livre de impurezas. Para eficiência deste sistema, entre outros cuidados, merece atenção especial a troca de filtro a cada 90 dias. Se este cuidado não for observado, a filtragem tornar-se-á inadequada, causando alteração na qualidade e na quantidade de ar que entra na incubadora devido o filtro estar saturado de sujidade. Ao ministrar aula sobre o uso da incubadora o docente deve enfatizar a importância desses conhecimentos, pois, é imprescindível que a enfermeira saiba como utilizar este equipamento.

Os dados sobre limite máximo de tempo para eliminação de mecônio pelo recém-nascido mostram que apenas $25,4 \%$ dos alunos sabem que esta deve ocorrer até 24 horas após o nascimento. Conforme TARALLI (1989) e RAMOS et al. (1978), a falta de eliminação do mecônio não deve ultrapassar as 24 horas de vida. A não ocorrência dessa eliminação nesse período pode sugerir existência de doenças do trânsito intestinal, tais como atresia e paralisia. Para verificação de imperfuração anal, o anus é obrigatoriamente examinado no primeiro exame do recém-nascido. Estes aspectos devem ser ressaltados durante as aulas, sobretudo por ser obrigação também da enfermagem avaliar a integridade do recém-nascido no momento do nascimento e períodos subseqüentes.

Em relação aos outros cuidados de rotina foram estudados cinco procedimentos, cujos resultados são apresentados a seguir:

Tabela 3 - Conhecimento dos alunos de Graduação em Enfermagem sobre outros cuidados de rotina ao recém-nascido a termo

\begin{tabular}{lccc}
\hline $\begin{array}{c}\text { Outros Cuidados } \\
\text { de Rotina }\end{array}$ & \multicolumn{3}{c}{ Freqüuência } \\
\hline \multicolumn{1}{c}{ A spectos } & Respostas & Absoluta & Relativas \% \\
\hline $\begin{array}{l}\text { Finalidade da adrinistração } \\
\text { da vitarnina K }\end{array}$ & certa & 305 & 85,5 \\
Meio de controle da infecção & certa & 311 & 14,5 \\
no bercáno & errada & 44 & 12,2 \\
SINAIS & & & \\
Frequência Cardiaca & certa & 309 & 91,5 \\
& errada & 29 & 8,5 \\
Frequiência Respiratóna & certa & 66 & 25,4 \\
& errada & 194 & 74,6 \\
VITAIS & & & \\
Termperatura & certa & 168 & 54,8 \\
& errada & 134 & 45,2 \\
\hline
\end{tabular}

Nota: A soma das freqüências absolutas de cada aspecto não totaliza 357 alunos (total da amostra) em vista de alguns não terem respondido todas as questões

Fonte: Alunos de Graduação em enfermagem dos Municípios de São Paulo e de Guarulhos 
Na Tabela 3, no que concerne à medida do conhecimento relativo à finalidade da administração da vitamina K, grande parte $(85,5 \%)$ dos alunos respondeu corretamente que é para prevenir a doença hemorrágica do recém-nascido. Consoante VAZ et al. (1989), a doença hemorrágica do recém-nascido é decorrente da deficiência de vitamina $K$ necessária para síntese hepática de fatores de coagulação do complexo protombínico. A deficiência da vitamina $\mathrm{K}$ é conseqüente à incapacidade do recémnascido manter seu estoque adequado durante os primeiros dias de vida.

A respeito do conhecimento teórico sobre o meio de controle de infecção no berçário, a grande maioria $(87,8 \%)$ da amostra deu resposta certa que é lavar as mãos e antebraços. Esperava-se que todos os alunos respondessem corretamente, já que este procedimento é abordado em várias disciplinas durante o curso. Porém não é de todo insatisfatório, tendo em vista que ainda lhes faltam um ou mais semestres para sua graduação, quando terão oportunidade de adquirir esses conhecimentos.

Em relação às questões sobre sinais vitais, verifica-se grande número $(91,5 \%)$ de respostas corretas:
100 a 160 batimentos por minuto para a questão sobre freqüência cardíaca normal do recém-nascido; pouco mais da metade $(54,8 \%)$ dos alunos respondeu acertadamente que a temperatura normal do recém-nascido a termo é de $36,5^{\circ}$ a $37,0^{\circ} \mathrm{C}$ e apenas um quarto $(25,4 \%)$ sabe corretamente que a freqüência respiratória é de 40 a 50 movimentos por minuto (ARMELLINI, 1991; RAMOS et al., 1978).

Ressalta-se nesse grupo de respostas a quantidade elevada $(74,6 \%)$ de alunos que tem conhecimento errôneo sobre a freqüência respiratória do recém-nascido a termo. Tal dado é preocupante visto que a avaliação adequada desta freqüência permite detectar, em tempo, algumas doenças respiratórias do recém-nascido. Para ESPIRIDIÃO (1989), uma vez estabelecida a respiração regular, a freqüência normal será de 40 movimentos por minuto, porém vários fatores podem alterá-la, como aumento da temperatura corporal, atividade física, aumento do metabolismo e mudanças na oxigenação.

$\mathrm{Na}$ Tabela 4 mostra-se o resultado da mensuração da percepção dos alunos quanto ao aproveitamento do ensino teórico de Enfermagem Neonatológica e a suficiência dos conteúdos recebidos.

Tabela 4 - Percepção dos alunos do Curso de Graduação em Enfermagem sobre o aproveitamento e a suficiência do ensino teórico de Enfermagem Neonatológica

\begin{tabular}{|c|c|c|c|}
\hline \multicolumn{2}{|l|}{ Percepção } & \multicolumn{2}{|c|}{ Freqüûncia } \\
\hline Aspectos & Respostas & Absoluta & Relativas $\%$ \\
\hline $\begin{array}{l}\text { Grau de aproveitamento do conteúdo } \\
\text { teórico } \\
\text { Suficiência da quantidade do conteúdo } \\
\text { teórico }\end{array}$ & $\begin{array}{c}\text { Ótimo } \\
\text { Bom } \\
\text { Regular } \\
\text { Mau } \\
\text { Péssimo } \\
\text { Sim } \\
\text { Não } \\
\text { Nãotenho opinião }\end{array}$ & $\begin{array}{r}62 \\
225 \\
61 \\
3 \\
3 \\
140 \\
168 \\
28\end{array}$ & $\begin{array}{r}17,5 \\
63,6 \\
17,3 \\
0,8 \\
0,8 \\
41,7 \\
50,0 \\
8,3\end{array}$ \\
\hline
\end{tabular}

Nota: A soma das freqüências absolutas de cada aspecto não totaliza 357 alunos (total da amostra) em vista de alguns não terem respondido todas as questões

Fonte: Alunos de Graduação em enfermagem dos Municípios de São Paulo e de Guarulhos

Pelos dados da Tabela 4, 81,1\% dos alunos consideraram seu aproveitamento ótimo e bom, no que diz respeito ao conteúdo teórico de Enfermagem Neonatológica recebido durante o curso.

Em relação à suficiência da quantidade do conteúdo teórico de Enfermagem Neonatológica ministrado, os alunos ficaram quase que igualmente divididos ou seja: 50,0\% acharam-no insuficiente e $41,7 \%$ consideraram-no suficiente.

\section{CONCLUSÕES}

Os resultados da variável conhecimento teórico de Enfermagem Neonatológica de alunos de Cursos de Graduação em Enfermagem permitiram concluir que, de um modo geral, os alunos apresentaram conhecimentos regulares a respeito dos cuidados imediatos, mediatos e outros cuidados de rotina, destacando-se melhor nível para os cuidados imediatos e nível insatisfatório para os conhecimentos sobre mecanismo da perda de calor; período de troca de filtro da incubadora; limite máximo de tempo para eliminação de mecônio e frequência respiratória.

Alguns aspectos como: finalidade do Boletim de Apgar, mecanismo da perda de calor; período de troca de filtro da incubadora, tempo limite para eliminação de 
mecônio e freqüência respiratória quantidade elevada de alunos anotou erradamente.

Considera-se este resultado inquietante já que o conhecimento errado dos aspectos pode conduzir a tomada de decisões inadequadas quanto à assistência imediata e mediata de algumas intercorrências ao recémnascido a termo.

\section{CONSIDERAÇÕES}

O ensino teórico de Enfermagem Neonatológica deve alicerçar-se em conteúdos que habilitem o aluno a assistir ao recém-nascido a termo com sólida base teóricocientífica. Para tanto, compete aos docentes adotarem métodos didáticos que possibilitem a apreensão e aplicação do conteúdo teórico no exercício da assistência ao neonato a fim de que esta tenha a eficácia esperada. Cabe-lhes também realizar discussões periódicas entre seus pares, alunos e enfermeiros para o planejamento e avaliação do programa de Enfermagem Neonatológica, criando alternativas que possam melhorar o processo ensino-aprendizagem.

No que diz respeito à pesquisa ora realizada, espera-se que propicie a reflexão e convicção de que muito se deve ainda fazer para equacionar o processo ensino-aprendizagem na área estudada. Acredita-se que os aspectos relativos ao nível insatisfatório encontrado no estudo, poderá ser melhorado se a prática estiver sempre fundamentada em conhecimento teórico, para tanto, o docente deve, continuamente, verificar a aplicação do conteúdo ministrado na execução dos cuidados, e caso o programa curricular não os contemple é imprescindível incluí-los para a eficácia da ação prática em enfermagem neonatológica dos alunos.

\section{NEONATAL NURSING: UNDERGRADUATE NURSING STUDENTS' KNOWLEDGE}

The present study was carried out at the Municipalities of São Paulo and Guarulhos with undergraduate nursing students and aimed at evaluating their knowledge regarding neonatal nursing and, if necessary, to contribute to the program reformulation. Thus, we would be able to improve nurses knowledge on the full-term new born care. In order to develop a structured questionnaire to be used as an instrument to measure the studied variable, aspects of the theoretical content related to the most common care procedures at the neonatal unit were selected. Authors found that students' theoretical knowledge on the theme was regular.

\section{ENFERMERÍA NEONATOLÓGICA: CONOCIMIENTO DE LOS ALUMNOS EGRESADOS DEL CURSO DE ENFERMERÍA}

El presente estudio fue realizado entre los alumnos egresados de los cursos de enfermería de las Municipalidades de São Paulo y Guarulhos, con el objetivo de evaluar sus conocimientos sobre la enfermería neonatológica y contribuir, si fuera necesario, para la re-formulación del programa. De esta forma, podríamos perfeccionar el conocimiento del enfermero sobre los cuidados del recién nacido dentro del plazo normal de gestación. Para elaborar un cuestionario estructurado, que fue utilizado como instrumento de medición de la variable, fueron seleccionados aspectos de contenido teórico concernientes a los cuidados más comunes en la unidad de neonatología. Se observó que el nivel de conocimiento teórico de los estudiantes sobre este tema era regular.

TÉRMINOS CLAVES: enfermería materno-infantil, educación

\section{ANEXO \\ CARTA DE APRESENTAÇÃO e TERMO DE CONSENTIMENTO}

Eu me chamo Masuco Naganuma sou enfermeira, estou fazendo uma pesquisa com alunos do curso de graduação egressos da disciplina de enfermagem neonatológica.

A pesquisa é sobre conhecimento, prática e percepção de alunos sobre enfermagem neonatológica. Tem como objetivo apresentar subsídios ao ensino de enfermagem neonatológica e, conseqüentemente, contribuir para melhoria da assistência de enfermagem ao recém-nascido.

Para a efetivação do estudo é imprescindível a participação dos alunos, respondendo ao questionário.
A fidedignidade do trabalho depende da sinceridade de suas respostas. Solicito, que ao responder as questões você seja franco. $\mathrm{O}$ questionário deve ser respondido seqüencialmente da primeira a última questão e sem leitura prévia de todas as questões. Os números colocados entre parênteses são códigos que serão utilizados pelo pesquisador.

Você não deve assinar o questionário, assim estará assegurando seu anonimato.

$\mathrm{Na}$ certeza de que você está prestando inestimável colaboração, agradeço antecipadamente.

Atenciosamente, 


\section{REFERÊNCIAS BIBLIOGRÁFICAS}

01. ARMELLINI, P.A. Procedimentos técnicos em neonatologia. In: SEGRE, C.A.M.; ARMELLINI, P.A.; MARINO, W.T. RN. 3.ed. São Paulo: Sarvier, 1991. p. 416-20.

02. CASANOVA, L.D. Artéria umbilical única. In: SEGRE, C.A.M.; ARMELINI, P.A.; MARINO, W.T. RN. 3.ed. São Paulo: Sarvier, 1991. p. 8991.

03. ESPIRIDIÃO, S. Filosofia do recém-nascido e sua adaptação ao meio extra-uterino. In: VIEGAS, D. VILHENA-MORAES, R. Neonatologia clínica e cirúrgica. São Paulo: Atheneu, 1989. p. 143-6.

04. MARINHO, E.L. Assistência ao recém-nascido aparentemente sadio. In: FONTES, J.A.S. Manual de perinatologia. Salvador: Fundo Editorial; BYK Procienx, 1981. p. 49-62.

05. PIZZATO, M.G.; POIAN, V.R.L.da. Enfermagem neonatológica. 2.ed. Porto Alegre: D.C. Luzzato, 1988. 162p.

06. RAMOS, J.L.A. Âmbito e finalidade da pediatria neonatal. In: ALCANTARA, P.; MARCONDES, E. Pediatria básica. 6.ed. São Paulo: Sarvier, 1978. v. 1, p. 353-4.

07. RAMOS, J.L.A.; CORRADINI, H.B.; VAZ, F.A.C. Cuidados ao recém-nascido normal e de baixo peso. Diagnóstico das condições do recémnascido. In: MARCONDES, E. Pediatria Básica. 6.ed. São Paulo: Sarvier, 1978. v. 1, p. 422-31.
08. SÃO PAULO. Secretaria de Estado da Saúde. Coordenadoria de Serviços Técnicos Especializados. Berçário de recém-nascido. São Paulo: Divisão de Saúde Materna e da Criança, 1976. 93p. (Publicação, 27).

09. SEGRE, C.A.M. Avaliação e exame do RN. In: SEGRE, C.A.M.; ARMELINI, R.A.; MARINO, W.T. RN. 3.ed. São Paulo: Savier, 1991. p. 34-7.

10. SEGRE, C.A.M.; MARINO, W.T. Atendimento ao RN na sala de parto. In: SEGRE, C.A.M.; ARMELLINI, P.A.; MARINO, W.T. RN. 3.ed. São Paulo: Sarvier, 1991. p. 24-31.

11. TARALLI, M. Exame físico do recém-nascido. Exame pediátrico. In: VIEGAS, D.; VILHENAMORAIS, R. Neonatologia clínica e cirúrgica. Rio de Janeiro: Atheneu, 1989. p. 149-60. v. 1.

12. VAZ, F.A.C.; RAMOS, J.L.A.; LEONE, C.R. Doença hemorrágica do recém-nascido e CIVD. In: VIEGAS, D.; VILHENA-MORAES, R. Neonatologia clínica e cirúrgica. Rio de Janeiro: Atheneu, 1989. v. 1, p. 573-6.

13. VIEGAS, D.; MONETTI, V. Conceitos e definições em Neonatologia: evolução da natalidade e mortalidade infantil em nosso meio, relativamente às outras áreas selecionadas. In: VIEGAS, D.; VILHENA-MORAES, R. Neonatologia clínica e cirúrgica. Rio de Janeiro: Atheneu, 1989. v. 1, p. 7-19.

14.WHALEY, L.F.; WONG, D. Enfermagem pediátrica: elementos essenciais a intervenção efetiva. (Essentials of pediatric nursing). 2.ed. Rio de Janeiro: Guanabara Koogan, 1989. 910p. 